\title{
What Effect Does Measuring Children under Anesthesia Have on the Blood Oxygenation Level-Dependent Signal? A Functional Magnetic Resonance Imaging Study of Visual Cortex
}

\author{
VALENTINE L. MARCAR, THOMAS LOENNEKER, ANDREA E. STRÄSSLE, \\ UWE SCHWARZ, AND ERNST MARTIN
}

\begin{abstract}
Department of Neuropsychology [V.L.M.], Psychological Institute, University of Zürich, CH-8032 Zürich, Switzerland; MR-Center, Department of Diagnostic Imaging [T.L., A.E.S., E.M.], University Children's Hospital Zürich, CH-8032 Zürich, Switzerland; and Department of Anaesthesiology [U.S.], University Hospital Zürich, CH-8091 Zürich, Switzerland
\end{abstract}

\begin{abstract}
We performed functional magnetic resonance measurements involving visual stimuli on 10 children. Half of the children were measured awake, the other half were measured under light Sevoflurane anesthesia corresponding to 0.5 mean alveolar concentration. Each child was presented with a flashed and a reversing checkerboard, which previous investigations have shown to induce identical increases in cerebral blood flow. The latter stimulus activated double the number of neurons as the former so that cerebral metabolic rate of oxygen consumption $\left(\mathrm{CMRO}_{2}\right)$ was doubled, leading to an effective rise of the oxygen extraction fraction. We measured the extent of activation by counting the number of activated pixels and assessed the change in $\mathrm{CMRO}_{2}$ by measuring the change in the local deoxyhemoglobin $(\mathrm{HbR})$ concentration, using change in spin relaxivity. In both groups of children, the extent of activation was larger for the flashed than the reversing checkerboard, although the absolute number of activated voxels was smaller for the children who were measured under anesthesia. The HbR concentration was significantly higher during the presentation of the reversing compared with the
\end{abstract}

\section{ABSTRACT}

flashed checkerboard. The relative change in the HbR concentration to the flashed and reversing checkerboard was the same in the children who were measured under anesthesia as in the children who were measured awake. We conclude that light levels of anesthesia may reduce the extent of activation but does not unduly influence either $\mathrm{CMRO}_{2}$ or cerebral blood flow, thus preserving the blood oxygenation level-dependent signal amplitude. (Pediatr Res 56: 104-110, 2004)

\begin{tabular}{|c|}
\hline $\begin{array}{l}\text { Abbreviati } \\
\text { BOLD, blood oxygenation level de } \\
\text { CBF, cerebral blood flow } \\
\text { CMRO }_{2} \text {, cerebral metabolic rate of } \\
\text { fMRI, functional magnetic resonan } \\
\text { HbR, deoxyhemoglobin } \\
\text { MAC, mean alveolar concentration } \\
\text { MRI, magnetic resonance imaging } \\
\Delta \mathbf{R} 2^{*} \text {, change in spin relaxivity }\end{array}$ \\
\hline
\end{tabular}

Our understanding of the neuronal mechanisms involved in sensory and cognitive processes has advanced considerably with the advent of modern imaging methods. Functional magnetic resonance imaging (fMRI) makes use of the blood oxygenation level-dependent (BOLD) signal to visualize brain activity. The BOLD signal relies on the paramagnetic properties of deoxyhemoglobin $(\mathrm{HbR})$ as a concentration-dependent,

Received June 17, 2003; accepted August 4, 2003.

Correspondence: Valentine L. Marcar, D.Phil., Department of Neuropsychology, Psychological Institute, University of Zürich, Treichlerstrasse 10, CH-8032 Zürich, Switzerland; e-mail: v.marcar@psychologie.unizh.ch

This work was supported by the Swiss National Foundation for Scientific Research (SNF Grant: 31-59363.99) and a grant from the Hartmann-Müller Foundation.

This study was presented at the ISMRM Annual Meeting 2003 in abstract form.

DOI: 10.1203/01.PDR.0000130479.43442.5C endogenous contrast agent. The increase in neuronal activity induced by a stimulus is quickly followed by a vascular response. Increasing neuronal activity leads to an increase in cerebral metabolic rate of oxygen consumption $\left(\mathrm{CMRO}_{2}\right)$, which in turn increases the rate at which oxyhemoglobin is converted to HbR. The increase in the local HbR concentration is offset by the vascular response, resulting in an increase in the magnetization signal, commonly referred to as the BOLD response.

The noninvasive nature of fMRI makes it an ideal tool for imaging brain activity in children to follow maturational changes and to obtain an insight into the development of the human CNS (1-4). Performing and interpreting functional imaging data of the immature brain presents scientists with two 
main difficulties. The first is the difficulty of finding a normal cohort. The second and perhaps more important difficulty is that children, particularly young children, more often than not have to be measured during natural or induced sleep $(2,3,5-8)$.

The influence on the BOLD signal of testing children under natural and induced sleep has been the subject of several investigations. The conclusions drawn from these unfortunately are still contradictory. Some reported that the incidence of negative BOLD responses increases during natural sleep $(2,5)$ or induced sleep $(9)$, suggesting an increase in the $\mathrm{HbR}$ concentration as a result of an attenuation of the vascular response. Others reported that the sedative agent reduces the neuronal activity $(6,7,10)$. The above studies have shown clear differences in the BOLD response during natural or induced sleep, but its origin is still uncertain. If fMRI measurements are to assist our understanding of the functional changes resulting from cerebral maturation, then it is necessary to determine which factors change during sleep and how these manifest themselves in the BOLD response.

We investigated whether the anesthetic agent Sevoflurane altered the BOLD response in visual cortex by attenuating the neuronal activity or the vascular response. The main obstacle that needed to be overcome was that the neuronal activity and the vascular response seem to be linked. Increasing the neuronal activity increases the cerebral blood flow (CBF), and vice versa. What was needed was a set of stimuli with either the identical levels of neuronal activity or identical CBF responses. In their seminal work, linking the $\mathrm{CBF}$ response in visual cortex to stimulus frequency, Fox and Raichle (11) used a flashed and reversing stimulus to overcome the confounding effects of stimulus frequency and mean stimulus intensity. On the CBF response of the flashed and reversing stimulus at the different frequencies, the authors concluded, "The response curves of the local blood flow change as a function of stimulus rate demonstrate the near identity of the regional response, despite differences between the two stimulus modalities in luminance, pattern, distance from retina and degrees subtended in the visual field." The flashed and reversing stimuli induce identical CBF responses in visual cortex.

We used a flashed and reversing checkerboard as shown in Figure 1. Both stimuli subtended the same visual angle and so projected on the same area of visual cortex. Because of the topographic organization of the visual cortex, the reversing checkerboard will activate all of the visual, cortical area onto which it projects, whereas the flashed checkerboard will activate only half of the area. Doubling the area of visual cortex being activated doubles the number of cells being activated, which in turn doubles the $\mathrm{CMRO}_{2}$. Both stimuli induced identical increases in CBF so that doubling the $\mathrm{CMRO}_{2}$ doubles the oxygen extraction fraction, which doubles the local $\mathrm{HbR}$ concentration. If the anesthetic agent Sevoflurane attenuates the neuronal activity more than the $\mathrm{CBF}$, then the local $\mathrm{HbR}$ concentration would decrease and the BOLD signal amplitude increase. If the neuronal activity is reduced less than the vascular response, then the local HbR concentration would increase and the BOLD signal amplitude decrease. The question that we aimed to address is whether the neuronal metabolic

\section{Flashed Reversed}

\section{Luminance Contrast}

\section{Chromatic Contrsat}

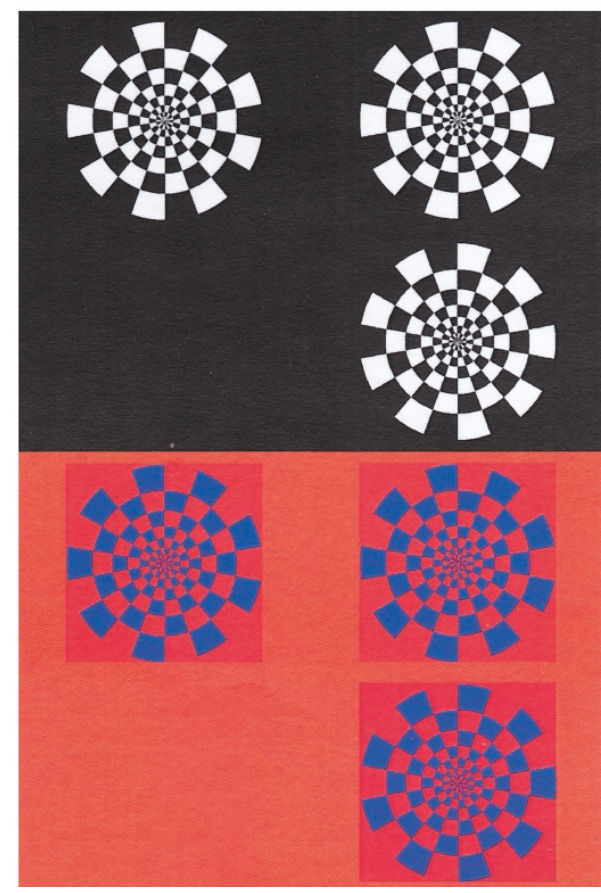

Figure 1. The four different checkerboard stimuli used in our investigation. ( $A$ and $C$ ) The flashed checkerboard. ( $B$ and $D$ ) The reversing checkerboard.

activity $\left(\mathrm{CMRO}_{2}\right)$ or the $\mathrm{CBF}$ is more strongly altered when children are measured during Sevoflurane anesthesia.

\section{METHODS}

Subjects. We performed fMRI on 10 children (five boys and five girls). The children who were measured under anesthesia were measured as part of a diagnostic MRI session that had to be performed under anesthesia because clinical indications necessitated it. Because these children usually displayed or were suspected of a medical condition warranting a diagnostic MRI, it was important to set strict inclusion and exclusion criteria. We excluded children with metabolic anomalies or micro-, macro-, or hydrencephaly, children had experienced hypoxic asphyxia, and children who had abnormalities of the visual system or had a tumor in the cortex located posterior to the central sulcus. Table 1 shows the clinical indications of the children who were measured under anesthesia in our study.

The children who were tested awake were recruited from a pool of normal, healthy children. The mean age of the children who were tested under anesthesia was 8.3 y (range, 7.1-10.2

Table 1. Clinical indicators that led to a diagnostic MRI being prescribed

\begin{tabular}{ll}
\hline Child measured under anesthesia & Clinical indicator \\
\hline A1 & Cranial fracture \\
A2 & Spinal MRI \\
A3 & Tumor in extremity \\
A4 & Spinal MRI \\
A5 & Tumor in medulla \\
\hline
\end{tabular}


y); that of the children who were tested awake was $9.1 \mathrm{y}$ (range, 6.7-11.6 y). The testing procedure was approved by the hospital's ethics committee. Informed parental and child consent was obtained in each case.

Anesthetic management. All anesthetic procedures were carried out by the hospital's anesthetist. The hospital guidelines on performing of MRI under anesthesia were adhered to at all times. Each patient was administered an oral dose of Midazolam (Dormicum, $0.5 \mathrm{mg} / \mathrm{kg}$; maximum dose of $10 \mathrm{mg}$ ). This is a short-acting sedative and is used routinely to prepare patients for anesthesia. Anesthesia was initiated using Sevoflurane administered via a breathing mask. Nitrous oxide was avoided so as not to have to contend with the influence of two anesthetic agents. Tracheal intubation followed. The muscle relaxant pancuronium bromide (Pavulon, $0.1 \mathrm{mg} / \mathrm{kg}$ ) was administered. Breathing and anesthesia were thereafter controlled via a mechanical ventilator. Throughout the entire MRI session, all vital signs including rectal body temperature, respiratory rate, heart rate, blood pressure, blood oxygen saturation, and expiratory $\mathrm{CO}_{2}$ were monitored constantly by the anesthetic team. Expiratory $\mathrm{CO}_{2}$ levels were kept between 4.8 and $6.0 \%$.

The depth of anesthesia is measured using the mean alveolar concentration (MAC). One MAC is defined as the concentration of a specific anesthetic agent at which half of the patients still respond to a noxious stimulus (e.g. surgical incision) (12). We performed our fMRI measurements with an anesthetic level of 0.5 MAC using the anesthetic agent Sevoflurane in an oxygen-air mixture. This level of anesthesia is considered light (12-14). In children who were measured under anesthesia, the eyelids were kept open using eyelid retractors made of titanium. Before applying the lid retractors, a short-term, local analgesic agent (Alocaine; Alcon Pharmaceuticals, Mumbai, India) was administered to the eye. This agent has no influence on the pupil or the lens. The conjunctiva and cornea were protected using a clear gel (Viscotears; Novartis, Switzerland). In applying the gel, we ensured that it sealed the eye and that it set to an even surface to minimize optical distortion of our image. We waited until the end-tidal, expiratory concentration of Sevoflurane corresponded to 0.5 MAC before commencing our fMRI measurements. In the time required for the anesthesia level to stabilize at $0.5 \mathrm{MAC}$, the clinic performed a diagnostic MRI measurement so as not to unduly delay the examination of the child. After the anesthetist signaled that the level of anesthesia was at $0.5 \mathrm{MAC}$ and stable, the functional measurements commenced. The fMRI measurements did not exceed $15 \mathrm{~min}$. At the end of the fMRI measurements, the eyelid retractors were removed and the protective gel was washed out. The eyes were then taped shut, as is customary for the institute when diagnostic MRI is being performed under anesthesia. The children who were tested awake were instructed to keep their eyes in the middle of the radial checkerboard and avoid large eye movements.

Apparatus. Measurements were performed on a 2 Tesla Bruker Tomikon S200 whole-body scanner (Bruker-Medical, Ettlingen, Germany) using a surface coil operated in cross-coil mode. This coil had been modified by affixing a surface-coated mirror to it in such a way that an image projected onto a translucent screen at the back of the magnet could be reflected to the subject's eyes. The coil was positioned so that it was most sensitive to signals from the occipital lobe. Inspecting Figure 1 reveals a signal intensity loss in the anterior part of the brain. This is the result of the signal loss caused by our use of a surface coil. The advantage of using a surface coil is that we had a higher sensitivity in the region of the visual cortex.

Functional imaging was performed using a single-shot, gradient-echo, EPI sequence with the following parameters: repetition time, $2000 \mathrm{~ms}$; effective echo time, $58 \mathrm{~ms}$; spectral width of $100 \mathrm{kHz}$; and a tip angle of 90 degrees. We also used a fat-suppression pulse with a $350-\mathrm{Hz}$ bandwidth. A package of 14 slices with a thickness of $4.5 \mathrm{~mm}$ and a gap of $1 \mathrm{~mm}$ was placed horizontally through the calcarine sulcus so that the lowest slice coincided with the base of the occipital lobe. We ensured that the slice package was positioned in such a way that the entire posterior occipital cortex, including striate cortex, was included. The field of view measured $25 \times 25 \mathrm{~cm}$ with a matrix size of $128 \times 64$ pixels, resulting in a spatial resolution of $2.0 \times 4.1 \mathrm{~mm}$. The EPI images were then reconstructed to a matrix size of $128 \times 128$ elements, including zero filling.

Stimuli. Our stimuli consisted of two checkerboard pairs as shown in Figure 1. Each radial checkerboard subtended a visual angle of 36 by 36 degrees at a viewing distance of $1.13 \mathrm{~m}$. We presented both a high-luminance contrast (black and white) and a low-luminance, high-chromatic contrast (red and blue) checkerboard pair.

Each checkerboard pair consisted of a flashed and a reversing checkerboard. The white elements of a checkerboard represented half of the area of the stimulus. All elements of the reversing checkerboard inverted their luminance contrast, whereas only half of the elements (the white elements) did so in the flashed checkerboard. We used a reversal frequency of 1 $\mathrm{Hz}$, with each reversal lasting $500 \mathrm{~ms}$. This is slower than the frequency used in other studies. In adults, a temporal frequency of $8 \mathrm{~Hz}$ leads to the largest $\mathrm{CBF}$ response $(11,15-17)$, whereas the maximum increase in $\mathrm{CMRO}_{2}$ has been observed at $4 \mathrm{~Hz}$ $(11,15-18)$. Our reason for choosing this frequency is that the lower temporal and spatial frequencies have a better response by the immature visual system (19-23).

The luminance of the stimulus elements was measured using a luminance metre (LS 110; Minolta, Osaka, Japan). The luminance of the white elements was $144 \mathrm{~cd} / \mathrm{m}^{2}$; that of the black background was $0.4 \mathrm{~cd} / \mathrm{m}^{2}$. The luminance contrast ratio was $365: 1$. The luminance of the blue elements was $5.4 \mathrm{~cd} / \mathrm{m}^{2}$; that of the red background was $29.3 \mathrm{~cd} / \mathrm{m}^{2}$. The luminance contrast ratio was 5.5:1.

Procedure. Each measurement started with 10 dummy scans, intended to bring the spin system into a steady state. These scans were immediately discarded. The actual measurements started with 10 control scans $(20 \mathrm{~s})$, during which the subject viewed a static image of the checkerboard stimulus. Ten stimulation scans ( $20 \mathrm{~s})$ followed, during which either the flashed or the reversing checkerboard was presented. This cycle of control and stimulation period was repeated five times. The presentation order of the stimuli was randomized between subjects. 
Data analysis. We used the commercial package MEDx 3.4 (Medical Numerics Inc., Sterling, VA, U.S.A.) to analyze our functional data. We followed the procedure described of Boynton et al. (24) and divided our data analysis into two independent steps. First, we located the activated picture elements or voxels using the standard approach for fMRI studies. A motion correction using the automated image registration (25) was applied. This was followed by an intensity normalization, which adjusted the mean of the data to a value of 1000 . Furthermore, a multilinear correction for baseline drift was applied. Activated voxels were determined using a crosscorrelation analysis. The activated voxels detected were then subjected to a Bonferroni correction to correct for multiple testing. The correction threshold was obtained by convolving the uncorrected threshold $(p=0.05)$ with the number of voxels in a mask restricting the correction to the subject's head. The $\mathrm{x}, \mathrm{y}$, and $\mathrm{z}$ coordinates of each corrected, activated voxel was noted. These coordinates were then used to extract the time series data. We identified those voxels located within the calcarine sulcus and selected only these for our analysis.

On the basis of the time series data, we determined the change in $\mathrm{HbR}$ concentration by calculating the change in the spin relaxivity $(\Delta \mathrm{R} 2 *)$ between the baseline and each time point $(26-29)$. We defined our baseline signal $\left(S_{b}\right)$ as the average, transverse magnetization signal of the 10 control scans of the first cycle. We calculated $\Delta \mathrm{R} 2 *$ using $\Delta \mathrm{R} 2 *=$ $(-1 / \mathrm{TE}) \times \ln \left(\mathrm{S}_{\mathrm{t}} / \mathrm{S}_{\mathrm{b}}\right)$, where TE is the echo time, $\mathrm{S}_{\mathrm{t}}$ is the mean transverse magnetization signal at a specific time point, and $\mathrm{S}_{\mathrm{b}}$ is the baseline signal (29). A $\Delta \mathrm{R} 2 *$ value of 0 is indicative of no change in the HbR concentration between the time point and the baseline. A positive $\Delta \mathrm{R} 2 *$ value is associated with a negative BOLD response as it indicates an increase in the concentration of $\mathrm{HbR}$ compared with the baseline. A negative $\Delta \mathrm{R} 2 *$ value is associated with a positive BOLD response as it indicates a decrease in the concentration of HbR from the baseline. The $\Delta \mathrm{R} 2 *$ amplitude is proportional to the change in $\mathrm{HbR}$ concentration from the baseline.

\section{RESULTS}

We examined the extent of activation as measured by the number of activated pixels and the change in the local concentration of HbR using the $\Delta \mathrm{R} 2 *$. Because of the number of subjects tested and because previous studies have shown that the luminance contrast and the chromatic contrast stimuli yielded the same result (30), we combined the data from the luminance and chromatic contrast stimuli. Unless otherwise stated, all statistical analyses were performed using SPSS 10 (SPSS Inc., Chicago, IL, U.S.A.). The data were analyzed using repeated measures of the general linear model with stimulus and state of consciousness as factors. For brevity, we provide only details of the statistical analyses that yielded a $p$ $\leq 0.05$. We included $\mathrm{ETA}^{2}$ as a measure of the size of the effect independent of sample size.

Positive BOLD response. Figure 2 depicts the location of the activated voxels as projected onto an anatomic MR image of a child measured awake and a child measured under anesthesia. We noted that the extent of activation was larger for the

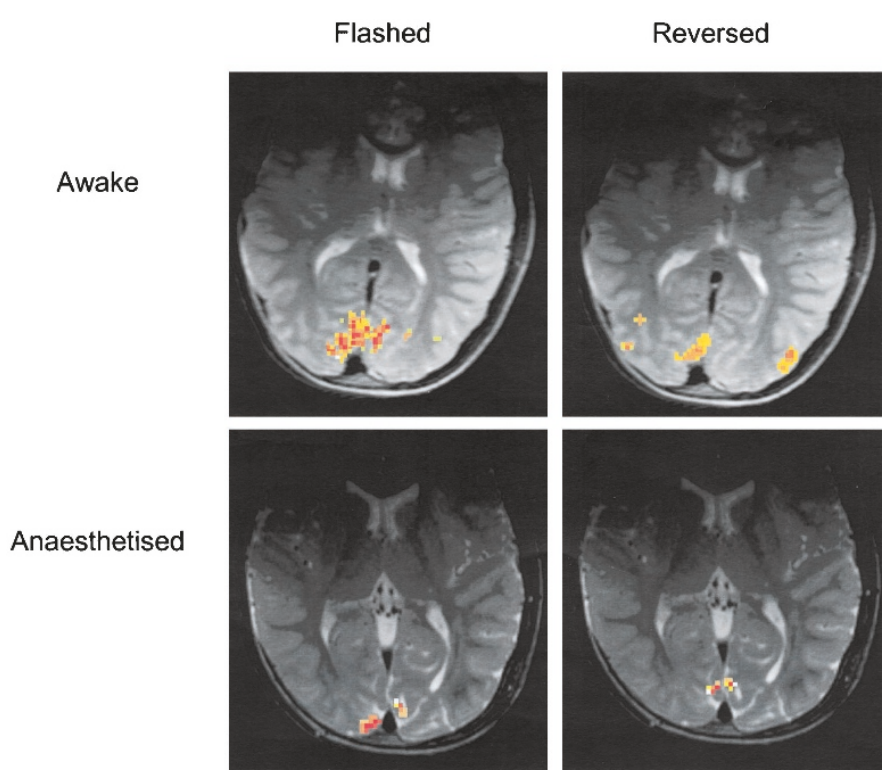

Figure 2. The location of the activated voxels to our flashed and reversing checkerboard stimuli in a child who was measured awake and a child who was measured under Sevoflurane anesthesia. The number of activated voxels may be smaller in the child who was measured under anesthesia, but it is still evident that the presentation of the flashed checkerboard induced a larger number of activated voxels than the reversing checkerboard.

flashed than the reversing stimulus $\left(F_{\text {Wilks }}=10.874, d f=\right.$ $\left.1,20, p=0.005, \mathrm{ETA}^{2}=0.437\right)$. There was a significant interaction between the state in which the children were tested $\left(F_{\text {Wilks }}=12.664 ; d f=1,20, p=0.003, \mathrm{ETA}^{2}=0.475\right)$.

The children who were measured awake yielded a larger number of activated voxels than the children who were measured under anesthesia. This is illustrated in Figure $3 \mathrm{~A}$. Examining the $\Delta \mathrm{R} 2 *$ for the flashed then the reversing checkerboard stimulus, we found a lower HbR concentration for the former than the latter $\left(F_{\text {Wilks }}=4.761, d f=1,12, p=0.05, \mathrm{ETA}^{2}=\right.$ $0.281)$. There was no significant interaction between the size of the $\Delta \mathrm{R} 2 *$ change and whether the children were tested awake and under anesthesia (Fig. $3 B$ ).

Negative BOLD response. We found no statistically significant differences in either the extent of activation (Fig. $4 A$ ) or the peak $\Delta$ R2* (Fig. $4 B$ ) between the children who were tested awake and under anesthesia. Finally, we found no statistically significant correlation between the number of activated voxels with a positive BOLD response and the number of activated voxels with a negative BOLD response.

\section{DISCUSSION}

Our study has shown that, although the extent of activation was affected considerably by the light level of Sevoflurane anesthesia used, the BOLD signal amplitude was not. If the BOLD signal amplitude was not altered, then neither the neuronal metabolic activity nor the vascular response could have been adversely influenced by light anesthesia. The results from fMRI under light anesthesia therefore are comparable to those obtained from the awake child. In the succeeding sections, we discuss some of the most important issues. 

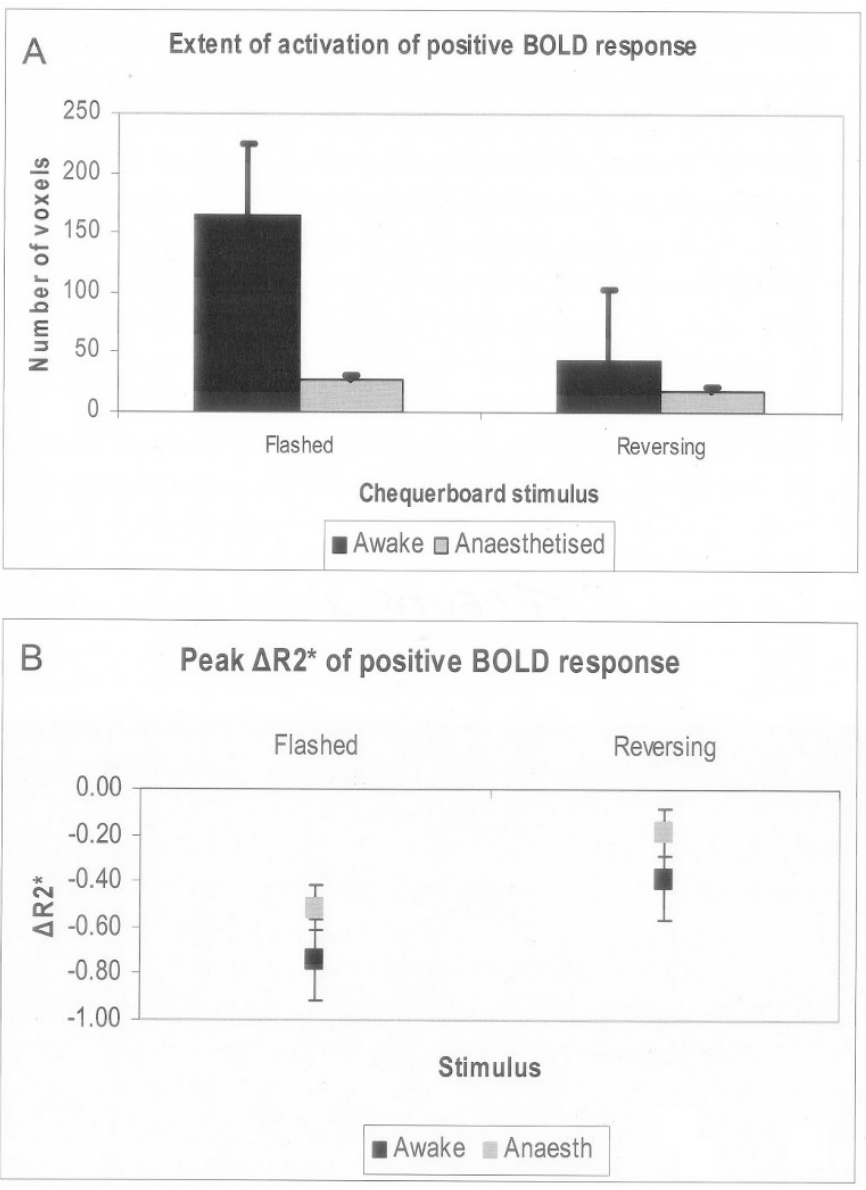

Figure 3. The mean number of activated voxels and the peak $\Delta \mathrm{R} 2 *$ for the positive BOLD response. $(A)$ The extent of activation using the mean number of activated voxels to the flashed and the reversing checkerboard. $\mathbf{\square}$, Children who were measured awake; $\square$, children measured during anesthesia. $(B)$ The peak $\Delta \mathrm{R} 2 *$ associated with the flashed and the reversing checkerboard stimulus. $\square$, Children who were measured awake; $\square$, children who were tested under anesthesia. The error bars indicate the SEM. The closer the mean peak $\Delta \mathrm{R} 2 *$ value is to 0 , the smaller the difference in the $\mathrm{HbR}$ concentration is compared with the baseline value.

Role of consciousness in visual processing. Because one group of children in our study was measured awake and the other under light anesthesia, it is important to examine the possible role of consciousness on our measurements. There is evidence that the lower visual areas are not entirely driven by the retinal input alone and that feedback projections also influence the neuronal activity. Our study revealed a close agreement in the BOLD signal amplitude between the awake and the anesthetized group to our stimuli. We interpret this as indicating that the BOLD response in our study was almost entirely due to the activation of a mechanism driven by the retinal input. Having a mechanism that is unaltered by consciousness may not provide any insight into the contribution of the cognitive development to visual perception. Our study instead provides a means of understanding the development of the hard-wired side of visual perception. Once this aspect is understood, the contribution made by the cognitive development in visual perception can be more readily distinguished from the more mechanical aspect.

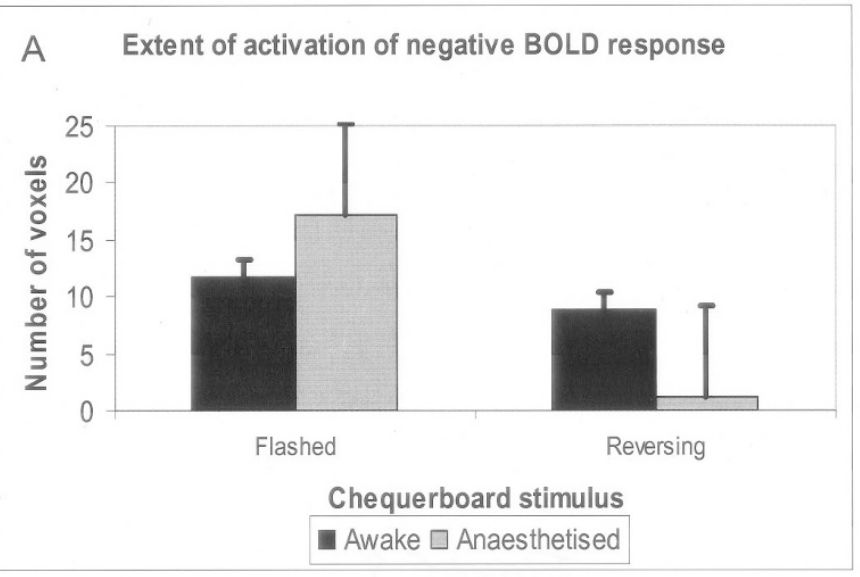

B

$$
\text { Peak } \Delta R 2^{*} \text { of negative BOLD response }
$$

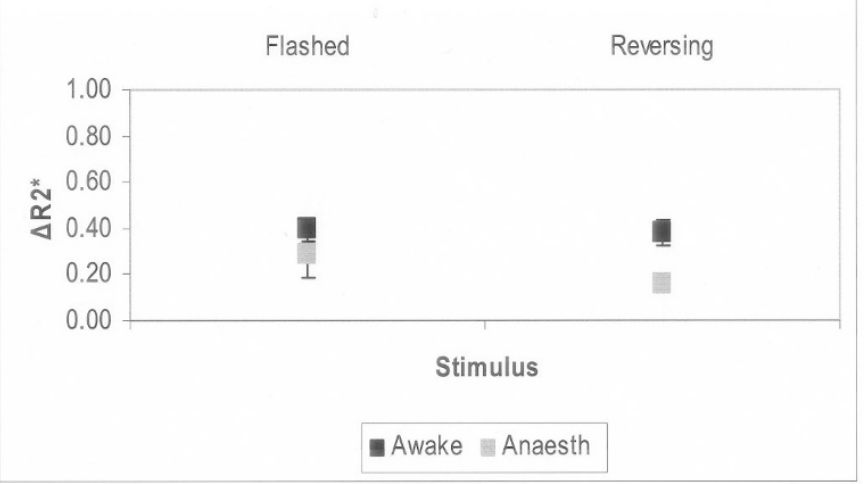

Figure 4. The mean number of activated voxels and the peak $\Delta \mathrm{R} 2 *$ for the negative BOLD response. $(A)$ The extent of activation using the mean number of activated voxels to the flashed and the reversing checkerboard. $\mathbf{\square}$, Children measured awake; $\square$, children who were measured during anesthesia. (B) The peak $\Delta \mathrm{R} 2 *$ associated with the flashed and the reversing checkerboard stimulus. $\square$, Children who were measured awake; $\square$, children who were tested under anesthesia. The closer the peak $\Delta \mathrm{R} 2 *$ value is to 0 , the smaller the difference in the HbR concentration is compared with the baseline value. The bars indicate the SEM.

Defining neuronal activity. Neuronal activity has traditionally been equated with the electrical discharge activity of neurons. fMRI using the BOLD signal detects changes in the $\mathrm{HbR}$ concentration within a region of cortex that can be as small as $1 \mathrm{~mm}^{3}$. This small volume, however, will contain millions of cells. The oxidative metabolism within this volume will depend not only on the electrical discharge activity of a group of cells but also and probably more so on the number of cells being activated.

Influence of our stimuli on the CBF. We were not able to include a $\mathrm{CBF}$ measurement for ethical reasons. Measuring the CBF response would have doubled the time required to complete our fMRI session or would have involved subjecting the children to a positron emission tomography measurement to obtain an accurate assessment of the CBF response (31). Instead, we relied on the published positron emission tomography data of Fox and Raichle (11), demonstrating that the CBF response to a flashed and a reversing stimulus is identical. Also, our finding that the $\Delta \mathrm{R} 2 *$ was larger for the flashed than 
the reversing checkerboard is consistent with the prediction by Buxton (32) for stimuli with identical CBF responses but different $\mathrm{CMRO}_{2}$ loads. These observations confirm that our checkerboard stimuli did induce identical CBF responses.

Neuronal activity and the BOLD response. In previous fMRI investigations, the electrical activity of a neuronal population has been manipulated by varying the stimulus contrast (26-28,33-36) or stimulus frequency $(11,15-18)$. In our study, the flashed and reversing checkerboards had the same luminance contrast and the same stimulus frequency. The neuronal electrical discharge activity associated with the two stimuli should therefore be identical. The reversing checkerboard, however, activated twice as many neurons as the flashed checkerboard. This enabled us to examine the influence of $\mathrm{CMRO}_{2}$ on the BOLD response. This being the case, the oxygen requirements resulting from an increase in the $\mathrm{CMRO}_{2}$ would have to be drawn from the identical blood volume. Increasing $\mathrm{CMRO}_{2}$ would increase the oxygen extraction fraction and so increase in the concentration of $\mathrm{HbR}$ in the venous blood.

We first consider the voxels with a positive BOLD response as these represent the standard BOLD response reported in most fMRI studies. Figure $3 B$ shows that the peak $\Delta \mathrm{R} 2 *$ was larger during the presentation of the flashed than the reversing checkerboard. This finding shows that the local HbR concentration reached a lower level during the flashed than during the reversing checkerboard. This is consistent with our view that the $\mathrm{CMRO}_{2}$ was lower during the flashed than during the reversing checkerboard stimulus, whereas the $\mathrm{CBF}$ response was identical for the two stimuli.

The negative BOLD response is harder to interpret as we found no difference in either the extent of activation (Fig. 4A) or the peak $\triangle \mathrm{R} 2 *$ (Fig. $4 B$ ) to the flashed and the reversing checkerboard. This suggests that the negative BOLD response is not related to either $\mathrm{CMRO}_{2}$ or $\mathrm{CBF}$ but to some other factor.

Influence of anesthesia on the positive BOLD response. We first discuss the extent of activation as indicated by the number of activated voxels. The children who were measured under light anesthesia yielded fewer activated voxels than the children who were measured awake. This indicated to us that anesthesia may have lowered the absolute number of activated voxels but that the relative difference between the stimuli was preserved. However, the flashed checkerboard still yielded the larger extent of activation than the reversing checkerboard in both groups of children (Fig. $3 A$ ). We found no difference in the peak $\Delta \mathrm{R} 2^{*}$, so the change in the local HbR concentration to our stimuli was the same for the children who were tested awake and under Sevoflurane anesthesia. This suggests that the anesthetic agent Sevoflurane at a concentration of 0.5MAC did not alter $\mathrm{CMRO}_{2}$ or interfere with $\mathrm{CBF}$.

A reduction in the extent of activation during anesthesia but a preservation of the $\mathrm{CMRO}_{2}$ and the $\mathrm{CBF}$ response reflects the idea that conscious processing depends on synchronized activity across several neuronal nets (37-42). The loss of consciousness results in a loss of synchronized activity across different neuronal nets, reducing the extent over which synchronized activation takes place while leaving the neuronal activity in the core net unaffected.
Influence of anesthesia on the negative BOLD response. The origin of the negative BOLD response is still not completely understood, but it is the result of an increase in the local $\mathrm{HbR}$ concentration. A number of studies have noted that the negative BOLD response increases in neonates compared with infants and young children (7), if subjects are measured during natural sleep $(2,5)$ or sleep resulting from the administration of a sedative agent $(6,9)$. We were interested in examining whether the light level of Sevoflurane anesthesia used in our study increased the incidence of a negative BOLD response. There was no difference in either the extent of activation or the $\mathrm{CMRO}_{2}$ between the children who were tested awake and under light anesthesia. The negative BOLD response does not seem to be influenced in any way by anesthesia. The difference in finding with respect to the negative BOLD response between our investigation and previous studies may be due to our having used an anesthetic agent (Sevoflurane) rather than a sedative agent.

Performing fMRI measurements under anesthesia. The extent of activation can best be thought of as the window through which we can measure the neuronal activation, whereas the peak $\Delta \mathrm{R} 2 *$ represents the neuronal metabolic activity. Provided that the window is not lost, the $\mathrm{CMRO}_{2}$ and the $\mathrm{CBF}$ are comparable to that seen in children who are measured awake. Performing fMRI measurements on children under Sevoflurane anesthesia will yield a BOLD signal amplitude that is a reliable indicator of the underlying neuronal activity. With the necessary care and precaution, it is possible to examine specific aspects of cerebral maturation using fMRI and compare the results between young children who were measured under light Sevoflurane anesthesia and older children who were measured awake. Whether this applies to other anesthetic agents and thus can be generalized will require further investigation.

\section{REFERENCES}

1. Anderson AW, Marois R, Colson ER, Peterson BS, Duncan CC, Ehrenkranz RA, Schneider KC, Gore JC, Ment LR 2001 Neonatal auditory activation detected by functional magnetic resonance imaging. Magn Reson Imaging 19:1-5

2. Born P, Rostrup E, Leth H, Peitersen B, Lou HC 1996 Change of visually induced cortical activation patterns during development. Lancet 347:543

3. Martin E, Marcar VL 2001 Functional MR imaging in pediatrics. Magn Reson Imaging Clin N Am 9:231-246

4. Thomas KM, Drevets WC, Whalen PJ, Eccard CH, Dahl RE, Ryan ND, Casey BJ 2001 Amygdala response to facial expressions in children and adults. Biol Psychiatry 49:309-316

5. Born AP, Miranda MJ, Rostrup E, Toft PB, Peitersen B, Larsson HB, Lou HC 2000 Functional magnetic resonance imaging of the normal and abnormal visual system in early life. Neuropediatrics 31:24-32

6. Martin E, Joeri P, Loenneker T, Ekatodramis D, Vitacco D, Hennig J, Marcar VL 1999 Visual processing in infants and children studied using functional MRI. Pediatr Res 46:135-140

7. Yamada H, Sadato N, Konishi Y, Kimura K, Tanaka M, Yonekura Y, Ishii Y 1997 A rapid brain metabolic change in infants detected by fMRI. Neuroreport 8:37753778

8. Yamada H 1997 [Brain functional MRI of the visual cortex with echo planar imaging]. Nippon Rinsho 55:1684-1687

9. Martin E, Thiel T, Joeri P, Loenneker T, Ekatodramis D, Huisman T, Hennig J, Marcar VL 2000 Effect of pentobarbital on visual processing in man. Hum Brain Mapp 10:132-139

10. Muramoto S, Yamada H, Sadato N, Kimura H, Konishi Y, Kimura K, Tanaka M, Kochiyama T, Yonekura Y, Ito H 2002 Age-dependent change in metabolic response to photic stimulation of the primary visual cortex in infants: functional magnetic resonance imaging study. J Comput Assist Tomogr 26:894-901

11. Fox PTR, Raichle ME 1985 Stimulus rate determines regional brain blood flow in striate cortex. Ann Neurol 17:303-305 
12. deJong RH, Eger EI 2nd 1975 AD50 and AD95 values of common inhalation anaesthetics on man. Anesthesiology 42:384-389

13. Eger EI 2nd, Saidman LJ, Brandstater B 1965 Minimum alveolar anesthetic concentration: a standard of anesthetic potency. Anesthesiology 26:756-763

14. Saidman LJ, Eger EI 2nd, Munson ES, Babad AA, Muallem M 1967 Minimum alveolar concentrations of methoxyflurane, halothane, ether and cyclopropane in man: correlation with theories of anesthesia. Anesthesiology. 28:994-1002.

15. Fox PT, Raichle ME 1984 Stimulus rate dependence of regional cerebral blood flow in human striate cortex, demonstrated by positron emission tomography. J Neurophysiol 51:1109-1120

16. Hagenbeek RE, Rombouts SA, van Dijk BW, Barkhof F 2002 Determination of individual stimulus-response curves in the visual cortex. Hum Brain Mapp 17:244250

17. Vafaee MS, Gjedde A 2000 Model of blood-brain transfer of oxygen explains nonlinear flow-metabolism coupling during stimulation of visual cortex. J Cereb Blood Flow Metab 20:747-754

18. Vafaee MS, Meyer E, Marrett S, Paus T, Evans AC, Gjedde A 1999 Frequencydependent changes in cerebral metabolic rate of oxygen during activation of human visual cortex. J Cereb Blood Flow Metab 19:272-277

19. Boothe RG 1982 Optical and neural factors limiting acuity development: evidence obtained from a monkey model. Curr Eye Res 2:211-215

20. Fiorentini A, Trimarchi C 1992 Development of temporal properties of pattern electroretinogram and visual evoked potentials in infants. Vision Res 32:1609-1621

21. Madrid M, Crognale MA 2000 Long-term maturation of visual pathways. Vis Neurosci 17:831-837

22. Tootle JS, Friedlander MJ 1989 Postnatal development of the spatial contrast sensitivity of X- and Y-cells in the kitten retinogeniculate pathway. J Neurosci 9:13251340

23. Zumbroich T, Price DJ, Blakemore C 1988 Development of spatial and temporal selectivity in the suprasylvian visual cortex of the cat. J Neurosci 8:2713-2728

24. Boynton GM, Engel SA, Glover GH, Heeger DJ 1996 Linear systems analysis of functional magnetic resonance imaging in human V1. J Neurosci 16:4207-4221

25. Woods RP, Cherry SR, Mazziotta JC 1992 Rapid automated algorithm for aligning and reslicing PET images. J Comput Assist Tomogr 16:620-633

26. Davis TL, Kwong KK, Weisskoff RM, Rosen BR 1998 Calibrated functional MRI: mapping the dynamics of oxidative metabolism. Proc Natl Acad Sci U S A 95:18341839

27. Hoge RD, Atkinson J, Gill B, Crelier GR, Marrett S, Pike GB 1999 Investigation of BOLD signal dependence on cerebral blood flow and oxygen consumption: the deoxyhemoglobin dilution model. Magn Reson Med 42:849-863
28. Kim SG, Rostrup E, Larsson HB, Ogawa S, Paulson OB 1999 Determination of relative $\mathrm{CMRO} 2$ from $\mathrm{CBF}$ and $\mathrm{BOLD}$ changes: significant increase of oxygen consumption rate during visual stimulation. Magn Reson Med 41:1152-1161

29. Ogawa S, Menon RS, Tank DW, Kim SG, Merkle H, Ellermann JM, Ugurbil K 1993 Functional brain mapping by blood oxygenation level-dependent contrast magnetic resonance imaging. A comparison of signal characteristics with a biophysical model. Biophys J 64:803-812

30. Bandettini PA, Kwong KK, Davis TL, Tootell RB, Wong EC, Fox PT, Belliveau JW, Weisskoff RM, Rosen BR 1997 Characterization of cerebral blood oxygenation and flow changes during prolonged brain activation. Hum Brain Mapp 5:93-109

31. Carroll TJ, Teneggi V, Jobin M, Squassante L, Treyer V, Hany TF, Burger C, Wang L, Bye A, Von Schulthess GK, Buck A 2002 Absolute quantification of cerebral blood flow with magnetic resonance, reproducibility of the method, and comparison with $\mathrm{H} 2(15) \mathrm{O}$ positron emission tomography. J Cereb Blood Flow Metab 22:11491156

32. Buxton RB 2002 Introduction to Functional Magnetic Resonance Imaging. Principles \& Techniques. Cambridge University Press, Cambridge, p 410

33. Boynton GM, Demb JB, Glover GH, Heeger DJ 1999 Neuronal basis of contrast discrimination. Vision Res 39:257-269

34. Goodyear BG, Menon RS 1998 Effect of luminance contrast on BOLD fMRI response in human primary visual areas. J Neurophysiol 79:2204-2207

35. Mohamed FB, Pinus AB, Faro SH, Patel D, Tracy JI 2002 BOLD fMRI of the visual cortex: quantitative responses measured with a graded stimulus at 1.5 Tesla. J Magn Reson Imaging 16:128-136

36. Schwarzbauer C, Heinke W 1999 Investigating the dependence of BOLD contrast on oxidative metabolism. Magn Reson Med 41:537-543

37. Dehaene S, Naccache L 2001 Towards a cognitive neuroscience of consciousness: basic evidence and a workspace framework. Cognition 79:1-37

38. John ER 2001 A field theory of consciousness. Conscious Cogn 10:184-213

39. Meador KJ, Ray PG, Echauz JR, Loring DW, Vachtsevanos GJ 2002 Gamma coherence and conscious perception. Neurology 59:847-854

40. Niebur E, Hsiao SS, Johnson KO 2002 Synchrony: a neuronal mechanism for attentional selection? Curr Opin Neurobiol 12:190-194

41. Taylor JG, Jancke L, Shah NJ, Nosselt T, Schmitz N, Himmelback M, Kalenscher T, Muller-Gartner HW 1998 A three stage model of awareness: formulation and initial experimental support. Neuroreport 9:1787-1792

42. von Arx WS 2001 On the biophysics of consciousness and thought and characteristics of the human mind and intellect. Med Hypotheses 56:302-313 